Herranz Llácer, C.V. (2018). Disponibilidad léxica de los futuros profesores de Educación Infantil y Primaria. Revista Electrónica Interuniversitaria de Formación del Profesorado, 21(1), 143-159.

DOI: http://dx.doi.org/10.6018/reifop.21.1.295271

\title{
Disponibilidad léxica de los futuros profesores de Educación Infantil y Primaria
}

\author{
Cristina V. Herranz Llácer \\ Universidad Rey Juan Carlos
}

\section{Resumen}

La investigación parte de analizar el léxico disponible de los alumnos/as de los Grados de Educación Infantil y Primaria matriculados en primer y cuarto curso, con objeto de valorar tanto su evolución como su mejora durante los cuatro años de formación universitaria. Para ello, se elaboró una prueba dividida en dos secciones: la primera recoge los datos sociodemográficos del estudiante y la segunda permitió evaluar el léxico disponible en torno a 18 Centros de Interés. Los resultados obtenidos identifican la existencia de un mayor léxico en los alumnos/as de cuarto curso que en los de primero, fundamentalmente en las áreas relacionadas con Educación. En este sentido, también se ha identificado la necesidad de reforzar otras áreas de conocimiento para conseguir una mejora del vocabulario global de los futuros docentes.

\section{Palabras clave}

Disponibilidad léxica; Educación infantil; Educación Primaria; Educación Universitaria.

\section{Lexical Availability in future teachers of Pre-Primary and Primary Education}

\section{Abstract}

The aim of this paper is analyzing the lexical availability of 591 students who are enrolled in the first and fourth courses of the pre-primary and primary education degrees. Additionally, the goal of this study is to analyze the evolution of the vocabulary during the four years of studies. A survey was created to develop both objectives, divided into two sections: the first one, which was centered on the sociodemographic data of the students, and a second

\section{Contacto:}

Cristina V. Herranz Llácer, cristina.herranz@urjc.es, Dpto. de Ciencias de la Educación, Lenguaje, Cultura y Artes, CC. Histórico-Jurídicas y Humanísticas y Lenguas Modernas, Universidad Rey Juan Carlos. Despacho 109, Departamental I, Camino del molino s/n. 28943 Fuenlabrada (Madrid) 
one, to assess the lexical availability through 18 centers of interest. The results show that there are variances between students of first and fourth course. In this sense, it is also important to do a reinforcement into the different areas of knowledge to improve the vocabulary of future teachers and to be able to obtain more significant differences.

\section{Key words}

Lexical availability; pre-primary education; primary education; higher education.

\section{Introducción}

Los trabajos de léxico disponible comenzaron en Francia a mediados del S. XX de la mano de Gougenheim, Michéa, Rivenc y Sauvageot (1964) con el objetivo de crear el lenguaje básico para ayudar en el proceso de enseñanza-aprendizaje del francés a los habitantes de las colonias francesas y a los inmigrantes que llegaban al país. El trabajo desarrollado por estos autores permitió comenzar con un nuevo campo de investigación que cuenta ya con una larga trayectoria. El Dr. López Morales está considerado como el principal representante de estos trabajos en español, gracias al Proyecto Panhispánico de Disponibilidad Léxica (PPHDL), que tiene como objetivo la realización de un diccionario de léxico disponible de los diferentes países de habla hispana.

El concepto de disponibilidad léxica fue definido por Michéa (1953 p. 310) como aquella palabra que "sin ser particularmente frecuente, está siempre lista para ser empleada y viene inmediatamente y naturalmente a la mente en el momento en el que se tiene necesidad de ella". Más tarde, a esta primera definición se añadió que además tenía que ser empleado en situaciones de diálogo precisas (López Chávez, 1994), de manera que pudiera explicarse que el léxico disponible contiene aquellas palabras que surgen en primer lugar y que se pueden usar de forma automática al hablar de un tema específico.

El objetivo de los estudios de disponibilidad léxica es recoger, analizar y valorar, cuantitativa y cualitativamente, el léxico disponible de una comunidad de habla específica (Ortolano Ríos, 2005). Así, de forma tradicional, los trabajos de disponibilidad léxica suelen estudiar el vocabulario de alumnos/as preuniversitarios nativos en torno a los denominados "centros de interés" (Bellón Fernández, 2011 o Martínez Olmos, 2015 entre otros). No obstante, cada vez son más frecuentes los trabajos orientados hacia otras realidades. En esta línea destaca la investigación de Santos Díaz (2015), que estudia el léxico disponible de alumnos/as del Máster en Formación del Profesorado; Bolaños Medina y Luján García (2010), que analizan los anglicismos de los estudiantes de Traducción e Interpretación; o Navarro Marrero (2009), que trabaja con un conjunto de estudiantes de Fisioterapia.

El concepto de centro de interés nació desde una doble visión: la primera, desde la pedagogía, donde Decroly utilizó esta idea para dar respuesta a las necesidades e intereses propios de sus estudiantes (Decroly, 2007); y, la segunda, desde la lexicografía y que fue descrita por Quemada (1967) en su estudio sobre los diccionarios de la lengua francesa moderna. En la disponibilidad léxica esta idea fue retomada por Gougenheim et al. (1964), que propuso dieciséis áreas asociativas: (1) Las partes del cuerpo, (2) La ropa, (3) Partes de la casa (sin los muebles), (4) Los muebles de la casa, (5) Los alimentos y las bebidas, (6) Objetos colocados en la mesa para la comida, (7) La cocina y sus utensilios, (8) La escuela: muebles y materiales escolares, (9) Calefacción y la iluminación, (10) La ciudad, (11) El campo, el pueblo o la aldea, (12) Medios de transporte, (13) Trabajos del campo y del jardín, (14) Los animales, (15) Juegos y distracciones y (16) Las profesiones (los diferentes oficios). 
En este trabajo, junto a las dieciséis áreas asociativas anteriores se han añadido dos centros de interés nuevos, (17) Nuevas Tecnologías: TIC y (18) Educación. El centro de interés 17 se ha incluido para recoger el léxico de una de las realidades más tangibles de los jóvenes actuales. Además, en relación con las Nuevas Tecnologías aplicadas a la educación, se puede afirmar que las TIC han llegado a las aulas para formar parte del proceso de enseñanza-aprendizaje (Gómez Gómez y García Aretio, 2016). Sin duda, resulta de especial interés conocer el léxico que manejan los futuros docentes sobre esta realidad. Por otro lado, el centro de interés 18 (Educación) se ha incluido para completar el área asociativa 8, centrada en objetos físicos de la escuela, porque lo que se busca en esta investigación es analizar las posibles diferencias en el léxico específico sobre Educación entre los alumnos/as de primero y cuarto de los Grados de Educación Infantil y primaria.

El uso de los centros de interés supone una ventaja fundamental porque los informantes evocan una lista de palabras que luego puede ser comparada (Murillo Rojas, 1993). Esto permite la contrastación no sólo de la información entre los sujetos de la misma investigación, sino con los de otros trabajos, ya que los estímulos (enunciados) son idénticos para todos y los criterios de edición del corpus -que se explicarán en el apartado de metodología- también lo son.

La aplicación de las pruebas de disponibilidad léxica -descrita por Gougenheim et al. (1964)- solicitaba a los informantes que escribieran un máximo de veinte palabras en torno a los dieciséis centros de interés anteriores. Unos años más tarde, Dimitrijévic (1969) cambió las listas cerradas por listas abiertas -i. e. ya no existe un límite de palabras para el listado- e incluye un tiempo máximo de respuesta: cinco minutos por áreas asociativa. Con estos cambios, aparentemente pequeños, se forma la base de los criterios actuales establecidos por el PPHDL. Las normas para la mayor parte de los trabajos de esta naturaleza cuentan con listas abiertas y con dos minutos de tiempo por centro de interés, aunque algunos autores como Velarde Inzunza (2014) emplean tres minutos. No obstante, los dos minutos se han establecido como un tiempo adecuado porque tras él es sabido que el léxico escrito deja de ser disponible (Ávila Muñoz, 2006) y entran en juego palabras que se deben al azar o a circunstancias particulares.

La disponibilidad léxica se puede analizar cuantitativamente mediante la fórmula matemática propuesta para este fin por López Chávez y Strassburger Frías (2000). Esta fórmula valora simultáneamente tanto la facilidad con la que un vocablo es producido como el orden de aparición y su frecuencia (Hernández Muñoz, 2015). Estas palabras (unidades léxicas) se recogen a través de pruebas asociativas, en las que el estímulo es el Centro de Interés, y cuya respuesta son las listas de vocabulario producidas por los sujetos evaluados. Así, los informantes transmiten las palabras que les vienen a la mente por cada centro de interés y que, por consiguiente, son aquellas que se activan de forma automática.

$$
\begin{aligned}
& \text { Donde: } \\
& D\left(P_{j}\right) \text { : Disponibilidad de la palabra } j \\
& \text { n: Máxima posición alcanzada en el centro de interés } \\
& D\left(P_{j}\right)=\sum_{i=1}^{n} e^{-2,3 \times\left(\frac{i-1}{n-1}\right)} \times \frac{f_{j i}}{I_{1}} \quad \begin{array}{l}
\text { i: Número de posición de que se trata } \\
\text { e: Número natural (2.718181818459045) }
\end{array} \\
& f_{j i}: \text { Frecuencia absoluta de la palabra j en la posición } i \\
& \mathrm{l}_{1} \text { : Número de informantes que participan en la encuesta }
\end{aligned}
$$

Figura 1.Fórmula de Disponibilidad Léxica. Fuente: López Chávez y Strassburger Frías (2000) 
Dado que el vocabulario es un aspecto vital para la enseñanza-aprendizaje de cualquier concepto o materia, sobre todo en los primeros años de la escolarización, y es una de las bases para la transmisión de conocimientos, esta investigación está centrada en el léxico disponible de estudiantes de los Grados en Educación (Infantil y Primaria) y las hipótesis surge tras detectar por parte de los profesores de los estudiantes algunas carencias en la competencia lingüística que afectan, fundamentalmente, a la falta de expresión y al vocabulario.

Con objeto de evaluar la situación actual a este respecto y, en consecuencia, poder implementar distintas medidas para suplir dichas carencias, el estudio se orientó a estudiantes de primero y cuarto curso. Concretamente, el objetivo final de esta investigación ha sido analizar si el léxico de los futuros docentes aumenta, disminuye o se mantiene en el momento de finalización de sus estudios con respecto al punto de partida. Está fuera de toda duda que su formación debe prepararlos consistentemente para la tarea que van a desempeñar (Faria, Reis y Peralta, 2016). Así, sin duda, deberían disponer de una adecuada formación lingüística para un apropiado ejercicio de su futura actividad laboral. Además, junto al análisis anterior, esta investigación propone emplear los estudios de disponibilidad léxica como una herramienta para la evaluación educativa de los estudiantes de áreas como la Educación Infantil y Primaria.

La consecución del objetivo final se ha articulado mediante el desarrollo de los siguientes objetivos específicos:

(1) La elaboración de los diccionarios de disponibilidad léxica, ya que permiten tanto conocer las palabras (repetidas o no) utilizadas por los informantes como comparar el léxico de los alumnos/as de primero y cuarto.

(2) La Identificación de los vocablos (palabras no repetidas) más disponibles para los informantes (alumnos/as) en cada uno de los centros de interés.

(3) La identificación de las áreas asociativas más abiertas y más cerradas mediante el cálculo del Índice de Cohesión.

(4) El análisis del aumento o disminución del léxico disponible para las áreas asociativas vinculadas de forma directa con la formación académica de los informantes (Centros de Interés 8 y 18).

(5) Conocer, si en el centro de interés 17 (Nuevas Tecnologías: TIC), los alumnos/as de cuarto curso presentan un léxico más relacionado con la Educación que los de primer curso.

\section{Metodología}

En primer lugar, se realizó un análisis descriptivo sobre las características sociodemográficas de los informantes con el objetivo de detectar posibles singularidades entre los alumnos/as participantes. Este aspecto se ha valorado como fundamental para interpretar de una manera más matizada los resultados posteriores.

En segundo lugar, a partir de la fórmula estadística propuesta por López Chávez y Strassburger Frías (2000) (vid. supra), se ha desarrollado el análisis del léxico disponible para los dieciocho centros de interés.

A continuación, se analizó el corpus completo compuesto por 205.097 palabras. El elevado número de unidades léxicas hizo que la edición de los materiales fuese una tarea difícil, en 
la que se trabajó de una manera lenta y detallada. Finalmente se obtuvieron 194.565 palabras. Resulta relevante destacar que este trabajo se realizó desde una perspectiva de mantenimiento de la información (Fernández Juncal, 2013), ya que de esta manera se mantienen los rasgos específicos del léxico de los informantes. Al mismo tiempo, se cumplen las normas actuales que fueron descritas por Samper Padilla (1998).

Hasta que Samper Padilla (1998) no elaboró los criterios de edición, éste era uno de los mayores problemas para los investigadores. A partir de su publicación se llegó a una serie de acuerdos que permiten poder comparar los distintos trabajos entre sí. A continuación, se especifican los criterios de edición que se han llevado a cabo para los dieciocho centros de interés:

- Se ha respetado el orden de aparición de las palabras dadas por los informantes.

- Tras la anotación en bruto de todas las palabras, se llevó a cabo la eliminación de las repeticiones dentro del mismo centro de interés y para un mismo sujeto.

- Se han utilizado los paréntesis ( ) para expresar la presencia o ausencia de ciertos elementos de una palabra. Por ejemplo, para la unidad léxica de abandono (escolar) se encontró que algunos de los sujetos sólo incluían abandono, mientras que otros escribieron abandono escolar (área asociativa 18 Educación).

- Al no poder discernir el grado de lexicalización de las marcas comerciales, se ha decidido seguir el criterio de Gómez Molina y Gómez Devís (2004), que incluyeron todas las marcas comerciales junto con un asterisco al final de la palabra.

- La unificación de la ortografía se hizo siguiendo los criterios expuestos en las siguientes obras: Real Academia Española (2014) y Real Academia Española y Asociación de Academias de la Lengua Española $(2005,2010)$.

- Los verbos son registrados en modo infinitivo.

- Los sustantivos se transcriben en singular y en su forma masculina.

\section{Instrumento}

El cuestionario realizado ad hoc para los informantes que fueron evaluados, estuvo compuesto por diez páginas (Anexo 1). En la primera página se encontraban las instrucciones de toda la prueba, que también fueron explicadas de forma verbal antes de comenzar. Las dos páginas siguientes estaban destinadas a recopilar la información sociodemográfica del estudiante. Tras esta primera parte, se incluyó una hoja en la que se pedía que no se continuara hasta recibir nuevas instrucciones. La última sección -realizada por todos los alumnos/as al mismo tiempo- contaba con seis hojas en las cuales había tres centros de interés en cada una de ellas, para que los alumnos/as pudieran contestar a la prueba de disponibilidad léxica. Los centros de interés analizados fueron los dieciocho mencionados anteriormente en el apartado de introducción.

\section{Procedimiento}

La prueba fue aplicada por una sola evaluadora en todos los grupos y respondida por los sujetos de forma voluntaria y anónima. Estos no recibieron ningún tipo de compensación por la tarea realizada.

La prueba se entregó de manera grupal y tuvo una duración de ejecución de sesenta minutos para cada grupo de alumnos/as. Este tiempo estaba distribuido de la siguiente forma: los primeros diez minutos eran empleados para la presentación y explicación de las instrucciones; a continuación, se dispuso de otros diez minutos para responder la primera parte destinada a los datos sobre el informante. Por último, se dieron dos minutos 
adicionales para completar cada uno de los dieciocho centros de interés. Pasado ese tiempo, los informantes debían pasar al centro de interés siguiente, sin poder volver al área asociativa anterior para evitar que el léxico recogido dejara de ser disponible. En esta última sección, destinada a los centros de interés, no se respondieron dudas por parte de los estudiantes para no influir en sus respuestas.

\section{Participantes}

De 614 sujetos evaluados, se analizaron los datos de 591 informantes matriculados en primero o cuarto del Grado de Educación Infantil o del Grado de Educación Primaria en la Universidad Rey Juan Carlos (Madrid). De estos sujetos, 129 son hombres (21.8\%) y 462 son mujeres (78.2\%).

El rango de edades de los informantes se sitúa entre los 17 y los 58 años, aunque la mayoría se encuentra entre los 17 y 24 años (537 sujetos que representan el 90.9\%) y tan sólo un 9.1\% se corresponde con sujetos mayores de 25 años ( 54 sujetos). La mayoría de los alumnos/as se encuentra en la edad normativa para estar cursando su primera titulación universitaria.

Gran parte de los estudiantes informantes (97.6\%) son de origen español. El resto de sujetos (2.4\%) procede de países como Rumanía - con una representación del 1.2\%-, Marruecos (0.5\%), Argentina (0.3\%), Uruguay (0.2\%) o Polonia (0.2\%). Este no resulta un dato sorprendente, porque el ratio de estudiantes universitarios que cursan sus estudios de forma completa en España es muy bajo (Michavila, Martínez, y Merhi, 2015).

Desde el punto de vista del origen, se puede deducir que el $98.1 \%$, correspondiente a 580 sujetos de los 591, ha especificado que su lengua materna es el español, mientras que tan sólo el $1.9 \%$ ha indicado que es el árabe, el polaco, el portugués o el rumano. No obstante, 10 de estos 11 alumnos/as correspondientes a ese $1.9 \%$ indican que tienen un nivel alto en el manejo de la lengua española. El sujeto restante presenta un nivel medio.

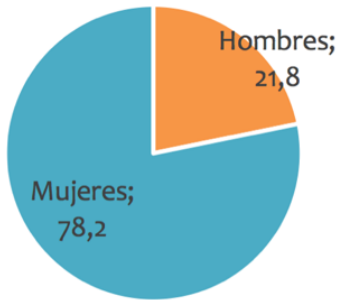

Figura 2. Sexo

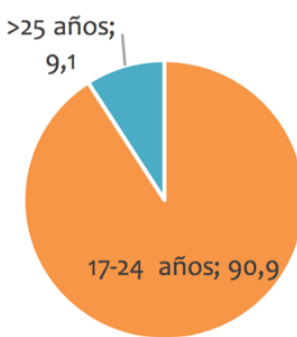

Figura 3. Sexo

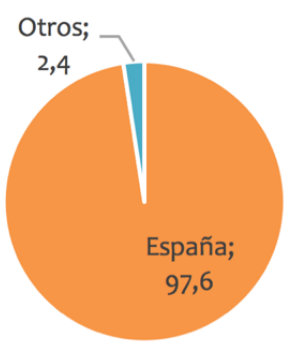

Figura 4. Procedencia

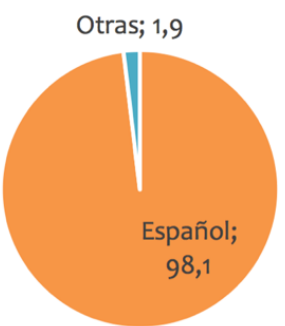

Figura 5. Lengua Materna

Respecto a la titulación en la que están matriculados, casi la mitad de los sujetos (48.4\%) está cursando el Grado de Educación Infantil y el 51.6\% el de Educación Primaria. Por otro lado, el $12.9 \%$ está estudiando en la modalidad bilingüe (inglés) frente al $87.1 \%$ que estudia en español. Esta diferencia resulta evidente al encontrarnos en España, y ser el español la lengua vehicular para la mayoría de los estudios. Además, la oferta para estudiar un grado en inglés es inferior que la de estudios en español. Asimismo, el $53.6 \%$ se encuentra en el primer curso de la titulación y el $46.4 \%$ en el último año. Este pequeño descenso se debe a los abandonos que sufren las titulaciones a lo largo de los diferentes cursos académicos.

Si se analiza el tipo de centro escolar de procedencia de los informantes antes de ingresar en la universidad, el $65.1 \%$ estudió en un centro público durante la educación secundaria frente a un $74.6 \%$ que lo hizo durante el bachillerato. El 34\% estudió en un centro privado o concertado durante la secundaria, mientras que en bachillerato desciende al $24.5 \%$. En 
España el porcentaje de alumnos/as de colegios concertados durante las etapas de primaria y secundaria es bastante elevado, aunque el número de estudiantes en los centros públicos sea superior. Sin embargo, este porcentaje disminuye durante el bachillerato porque la mayoría de los centros no disfrutan del concierto para esta etapa educativa (Secretaría general técnica y Subdirección general de estadística y estudios, 2015).

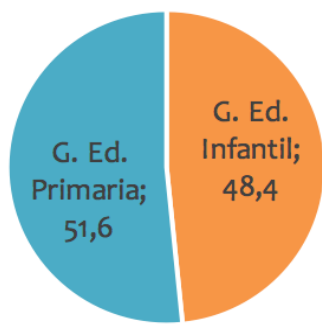

Figura 6. Titulación

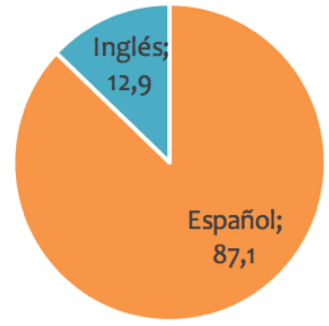

Figura 7. Lengua de Estudios

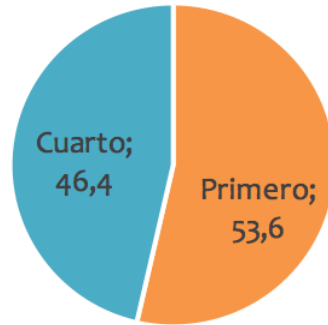

Figura 8. Curso

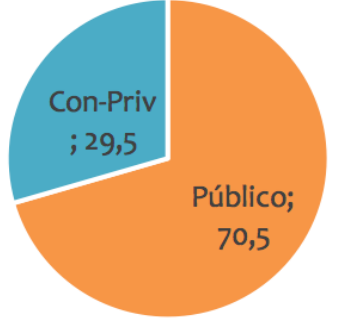

Figura 9. Centro escolar de procedencia

De los datos recogidos se extrae que un $42.1 \%$ de los informantes ha señalado que antes de ingresar en la universidad han estudiado Programas de Cualificación Profesional (PCPI), Formación Profesional (FP) de grado medio y/o de grado superior, representando según los estudios: PCPI (0.3\%), FP de grado medio (4.1\%) y FP de grado superior (37.7\%). Las titulaciones mayoritarias escogidas para FP de grado superior fueron Técnico Superior de Educación Infantil -con un porcentaje de 48.9\%- y la de Técnico de Animación de Actividades Físicas y Deportivas (TAFAD) con un $28.7 \%$. El $22.4 \%$ restante ha estudiado diferentes titulaciones como animación sociocultural, gestión de alojamientos turísticos, integración social, etc. Los alumnos/as que estudiaron Técnico Superior de Educación Infantil se matriculan en su mayoría en el Grado de educación Infantil y los de TAFAD en el Grado de educación Primaria con el objetivo de realizar la mención en educación física.

Además, si se divide al conjunto de alumnos/as informantes por el nivel socioeconómico, se identifica cómo el $19.8 \%$ de la muestra (117 sujetos) es de clase alta, el $69.2 \%$ (409 sujetos) de clase media y el 11\% (65 sujetos) de clase baja. Esto muestra una distribución acorde a la situación demográfica de nuestro país, donde la mayoría de la población pertenece a una clase media. El nivel socioeconómico se calculó relacionando el nivel de estudios de los padres, su actividad laboral así como el lugar y tipo de centro de estudios de los alumnos/as.

Finalmente, si se observa el nivel de estudios de los progenitores, se encuentra que un $3.4 \%$ de las madres y un $6.4 \%$ de los padres no tiene ningún tipo de estudios. Un $24 \%$ de las madres y un $21.8 \%$ de padres han completado los estudios primarios, y un $20.8 \%$ de las madres y un $20.6 \%$ de los padres han superado la secundaria. Con respecto a la Educación en otros niveles, un $11.8 \%$ de las madres y un $13 \%$ de los padres han completado los estudios equivalentes al bachillerato y el $19.5 \%$ de las madres y el $19.1 \%$ de los padres han completado algún tipo de Formación Profesional. Únicamente un $20.5 \%$ de las madres y el 19\% de los padres tienen estudios universitarios, por lo que se deduce que, para un elevado porcentaje de padres y madres, sus hijos/as son los primeros en llegar a la universidad dentro de su núcleo familiar. 


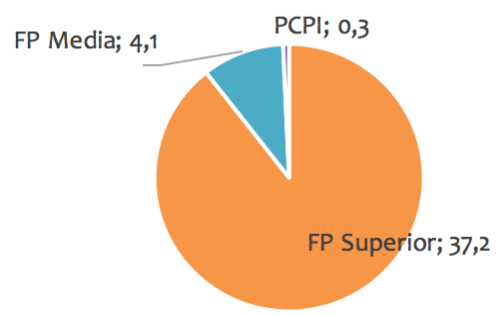

Figura 10. Estudios previos (PCPI, FP medio y FP superior)

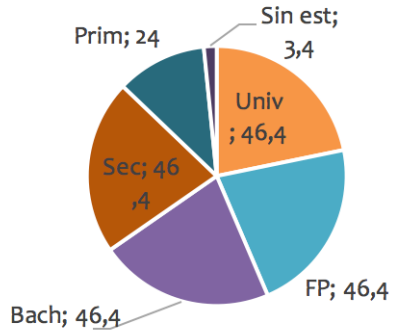

Figura 12. Nivel de estudio de la madre

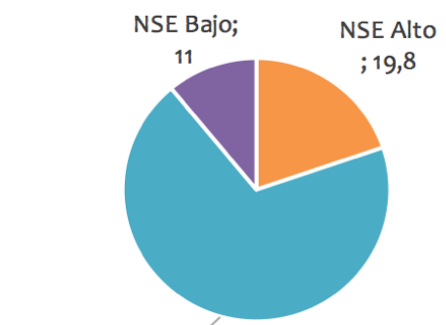

NSE Medio; 69,2

Figura 11. Nivel socioeconómico

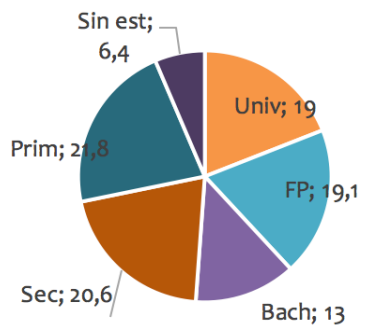

Figura 13. Nivel de estudio del padre

\section{Resultados}

\section{Resultados globales: datos generales para el total de los informantes y el corpus completo}

El número total de palabras para los 591 informantes y para los dieciocho centros de interés es de 194.565, que corresponde a una media de 329.2 unidades léxicas por sujeto y de 18.3 palabras de media para cada uno de los centros de interés. El número de palabras únicas recogidas entre todas las pruebas registradas es de 10.635. A pesar de presentar un corpus mayor que en el PPHDL y una media mayor por informante, los alumnos/as universitarios evaluados presentan una media ligeramente inferior por informante y centro de interés si se tienen en cuenta los dieciocho centros de interés, pero considerando los dieciséis clásicos la media está por encima en un 0.3.

Tabla 1.

Resultados globales

\section{Total Media por Media por Centro Media por Centro de}

palabias informante deinterés interés einformante

Disponibilidad léxica en alumnos/as

de educación

$194.565 \quad 329.2$

$10.809,2$

$18.3 \rightarrow 18$ centros de interés

Resultados Proyecto Panhispánico

(Fernández Juncal, 2013, P.49)
$98.721 \quad 296.46$

$6.170,1$
$18.8 \rightarrow 16$ centros de interés

18.5 
Del total de 194.565 palabras, se observa la existencia de grandes oscilaciones: desde 16.076 palabras que corresponden al $8.3 \%$ del total del corpus, en el centro de interés (14) Los animales a las 5.416 (2.8\% del total) en el centro de interés (13) Trabajos del campo y del jardín. De las dieciocho áreas asociativas, aparecen cuatro por encima de las trece mil palabras: Las partes del cuerpo, La ropa (de hombres y mujeres), Los alimentos y las bebidas y Los animales.

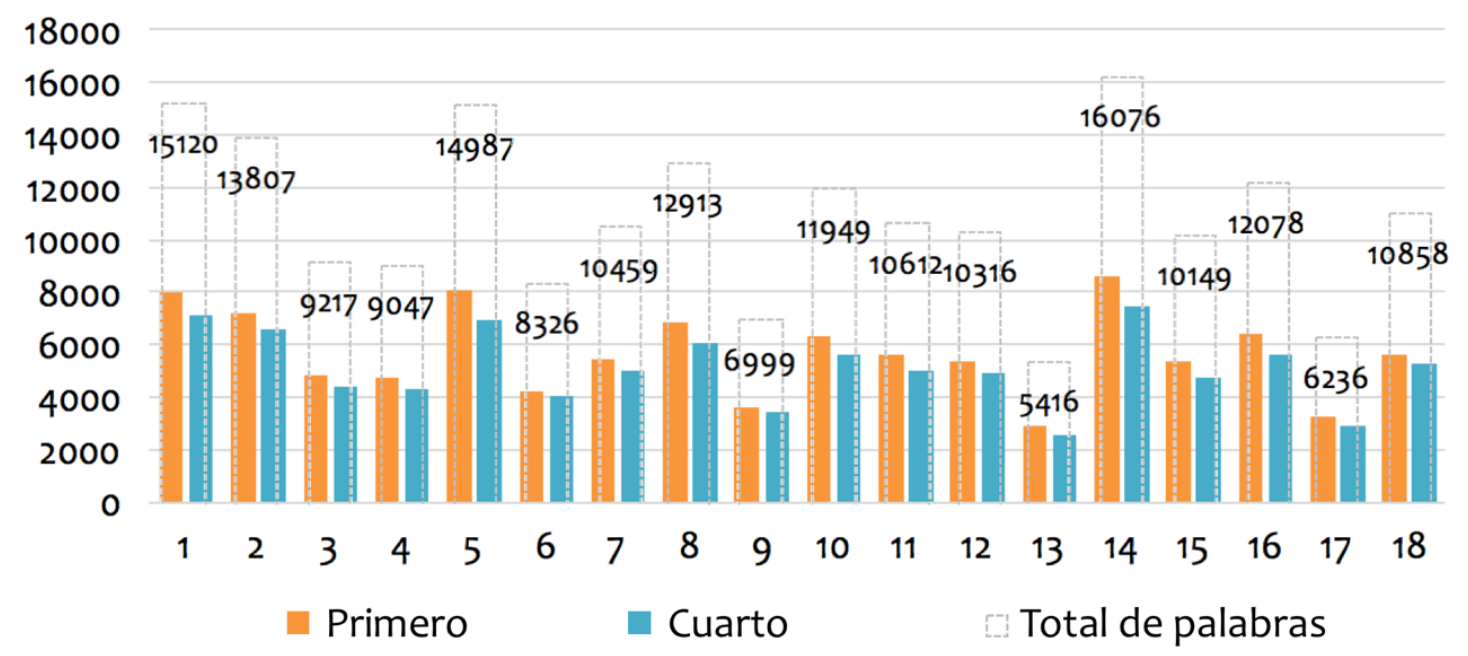

Figura 14. Número de Palabras por Centro de Interés para el total de la muestra

Entre las 10.000 y las 12.999 palabras localizamos los centros de interés (7) La cocina y sus utensilios, el (8) La escuela: muebles y materiales escolares, el (10) La ciudad, el (11) El campo, el pueblo o la aldea, el (12) Medios de transporte, el (15) Juegos y diversiones, el (16) Profesiones y oficios y el (18) Educación. Por debajo de las diez mil palabras quedarían los seis centros de interés restantes.

Como se ha podido comprobar, de un centro de interés a otro hay una gran variación. Esta diversidad identificada entre las distintas áreas asociativas proviene de si los informantes se sienten o están más o menos capacitados para incluir vocablos. Quizás, por este motivo, los centros de interés con una alta producción léxica como el número (14) Los animales, que presenta una media de 27.2 palabras frente a las 9.2 palabras en el centro de interés número (13) Trabajos del campo y del jardín.

Si se comparan estos resultados con los que se han obtenido en otras investigaciones, se encuentra que -una vez más- el área asociativa 1 está entre las primeras posiciones y la 9 entre las últimas (Bellón Fernández, Samper Hernández, y Samper Padilla, 2003). Esto puede confirmar que el propio enunciado (estímulo) influye en la producción lingüística de los informantes.

\section{Resultados al considerar el curso académico:}

A continuación, se recoge la descripción de los resultados obtenidos teniendo en cuenta la variable curso académico. Al no usar una variable clásicas para estos estudios (sexo, tipo de centro escolar, nivel socioeconómico o localización -rural/urbano-) no resulta posible comparar estos datos con los de investigaciones previas. 
Por un lado, de los 317 alumnos/as que están cursando primero, encontramos un total de 102.456 palabras, lo que corresponde a 323.2 palabras para las dieciocho áreas asociativas y una media por centro de interés de 18 palabras. Por otro lado, para los 274 estudiantes de cuarto tenemos un corpus de 92.109 palabras y unos valores medios algo superiores: 336.2 palabras de media para el total y 18.7 palabras por centro de interés. Valorando el objetivo final perseguido, esto indica una pequeña mejora del léxico de los alumnos/as de cuarto curso en comparación con los de primer curso -teniendo en cuenta el corpus completo-.

Si nos detenemos en cada uno de los centros de interés, tan sólo en un área asociativa los alumnos/as de primer curso obtienen una mayor puntuación que los de cuarto (en el número (5) Los alimentos y las bebidas), mientras que, para los diecisiete centros de interés restantes, en once de ellos los estudiantes de cuarto obtienen una diferencia en media superior a media palabra $-\mathrm{y}$, de estos, seis presentan una diferencia en media mayor o igual a la unidad-. Estos datos confirman que, en los valores medios, los alumnos/as de cuarto obtienen mejores resultados que los de primer curso.

Destaca especialmente el centro de interés (18) Educación, donde los estudiantes de cuarto curso superan con 1.4 palabras a los de primero. Este resultado puede deberse al bagaje que han ido adquiriendo los informantes de la muestra a lo largo de sus años de estudio y refuerza una de las preguntas iniciales de este trabajo: conocer si los informantes de cuarto obtenían mejores resultados léxicos que los de primero en relación con los centros de interés que mantienen afinidad directa con su formación.

Si se tiene en cuenta que los informantes que estamos analizando -en su mayoría- son "nativos digitales", resulta sorprendente que el centro de interés (17) Nuevas Tecnologías: TIC sea uno de los menos productivos. Finalmente, los centros de interés con mayor y menor número de palabras son el (14) Los animales, (5) Los alimentos y las bebidas y (1) Las partes del cuerpo. Mientras que los más productivos son el (12) Medios de transporte, (17) Nuevas tecnologías: TIC y (9) Calefacción e iluminación.

Por otro lado, el análisis de las palabras únicas (vocablos) presenta una serie de matices. Así, del corpus total se eliminan las repeticiones de palabras aportada por distintos informantes. Resulta sorprendente que, desde la perspectiva de la proporción (para evitar que la diferencia de tamaños poblacionales influya), los resultados entre primero y cuarto sean semejantes. ¿Puede esto significar que en realidad no hay diferencia-en relación con la disponibilidad léxica- entre los alumnos/as que comienzan y finalizan sus estudios a pesar de que para el total de las palabras si existían dichas diferencias?

Esta pregunta surge porque en nueve (de dieciocho) centros de interés los alumnos/as de primero superan a los de cuarto: (2) La ropa (de hombres y mujeres), (3) Las partes de la casa (sin los muebles), (4) Los muebles de la casa, (7) La cocina y sus utensilios, (10) La ciudad, (11) El campo,el pueblo o la aldea, (12) Medios de transporte, (15) Juegos y diversiones y Profesiones y oficios y tan sólo en cuatro: (1) Las partes del cuerpo, (5) Los alimentos y las bebidas, (8) La escuela: muebles y materiales escolares y (18) Educación son los de cuarto curso los que obtienen una mayor puntuación, mientras que en el análisis anterior-sobre el total de las palabras- los de primer curso superaban a los de cuarto solo en un centro de interés.

Tratando de dar respuesta a la pregunta anterior es posible afirmar que para las palabras únicas, no existe una diferencia clara entre los alumnos/as de primero y cuarto curso. Este resultado confirma -en cierta manera- que la formación inicial de los docentes no es suficiente (Ramírez García, 2015). No obstante, en los centros de interés relacionados con sus estudios, los alumnos/as de cuarto obtienen una mayor proporción que los de primero. Esto muestra que sí existe actualización del léxico entre los alumnos/as de primero y cuarto 
curso, al menos para aquellos centros de interés que mantienen una relación directa con sus estudios.

A continuación, se analizan los índices de cohesión y densidad léxica (Tabla 2) para poder conocer el nivel de coincidencia que existe en las respuestas de los informantes en un centro de interés (Hernández Muñoz, 2006). Con estos análisis, se pone en relación el número de palabras únicas y el número de palabras totales en cada centro de interés con el objetivo de determinar el grado de cohesión. Así, a un mayor índice de cohesión en un área asociativa implica que es más compacta o cerrada y viceversa. De esta forma, se puede conocer si las respuestas son más o menos homogéneas. En relación con estos índices, los alumnos/as de primero y cuarto obtienen -de nuevo- resultados semejantes para las áreas asociativas que muestran más variación, tratándose de los centros de interés (11) El campo, el pueblo o la aldea y el (18) Educación para ambos cursos.

No obstante, donde sí se identifican diferencias es en los centros de interés más densos o cerrados. Mientras que los centros de interés más compactos para los alumnos/as de primero son el (5) Los alimentos y las bebidas y el (1) Las partes del cuerpo. Para los de cuarto son el (2) La ropa (de hombres y mujeres) y el (4) Los muebles de la casa. Además, es importante señalar que los centros de interés 8 y 18 (que mantienen relación con su formación) presentan resultados semejantes siendo ambos más dispersos para los de cuarto curso que para los de primero.

Finalmente, pasando a observar tan solo los cinco vocablos (palabras únicas) más disponibles para cada uno de los centros de interés y por curso académico, se presentan los diccionarios (Tabla 3) de disponibilidad con el índice de disponibilidad léxica en porcentaje, tal y como recomienda Bartol Hernández (2017), dado que permite entender de forma más sencilla su valor. Como aspectos reseñables destaca:

- Coincidencias en los diccionarios:

- Los centros de interés $3,4,6,7,8,11,12,13,14,16,18$ coinciden en las cinco palabras más disponibles. De estos, el 3, 13, 14 y 18 también coinciden en el orden de aparición de las mismas.

- Los centros de interés: 1, 2, 5, 9, 10 y 17 presentan similitud en cuatro vocablos.

- El centro de interés 15 coincide en tres unidades léxicas.

En el centro de interés (5) Los alimentos y las bebidas, los estudiantes de cuarto presentan una marca comercial menos, desapareciendo Fanta* e incorpora la palabra zumo en su lugar, quedando registrado sólo Coca-Cola*. El hecho de disminuir el número de marcas comerciales denota una mejora del léxico.

Para el centro de interés (8) La escuela: muebles y materiales escolares nos resulta especialmente reseñable que los alumnos/as de cuarto escribieran en primer lugar mesa mientras que, para los de primero, su vocablo más disponible fue silla. Además, los alumnos/as de cuarto presentan la palabra lápiz en una posición superior a la de bolígrafo. El hecho de usar mesa y lápiz en primer lugar nos muestra el cambio de rol mental que los alumnos/as de cuarto han comenzado a realizar en relación con su próximo cambio de status a profesor, debido a que la silla se asocia directamente al alumno/a que debe permanecer sentado y el lápiz a los estudiantes de infantil y primaria que lo emplean de forma predominante frente al bolígrafo por el proceso de la iniciación de la escritura. 
- En el centro de interés (17) Nuevas Tecnologías: TIC mientras los alumnos/as de primero escriben la palabra IPhone*, los de cuarto presentan la palabra pizarra digital, que muestra -de nuevo-, una relación mucho más directa con su posterior trabajo.

Tabla 2.

Resumen de resultados

\begin{tabular}{|c|c|c|c|c|c|c|c|c|c|c|c|c|}
\hline & \multicolumn{4}{|c|}{ Total de palabras } & \multicolumn{4}{|c|}{ Total de palabras únicas } & \multicolumn{4}{|c|}{ Densidad Léxica e Índice de cohesión } \\
\hline & \multicolumn{2}{|c|}{ Primero } & \multicolumn{2}{|c|}{ Cuarto } & \multicolumn{2}{|c|}{ Primero } & \multicolumn{2}{|c|}{ Cuarto } & \multicolumn{2}{|c|}{ Primero } & \multicolumn{2}{|c|}{ Cuarto } \\
\hline & Pal. & Prom. & Pal. & Prom. & $\begin{array}{c}\text { Pal. } \\
\text { únicas }\end{array}$ & $\%$ & $\begin{array}{l}\text { Pal. } \\
\text { únicas }\end{array}$ & $\%$ & $\begin{array}{l}\text { Den. } \\
\text { Léx. }\end{array}$ & $\begin{array}{c}\text { Índ. } \\
\text { Cohesión }\end{array}$ & $\begin{array}{l}\text { Den. } \\
\text { Léx. }\end{array}$ & $\begin{array}{c}\text { Índ. } \\
\text { Cohesión }\end{array}$ \\
\hline 1. & 8.025 & 25.3 & 7.095 & 25.9 & 306 & 3.9 & 303 & 4 & 26.2 & 0.0827 & 23.4 & 0.0855 \\
\hline 2. & 7.231 & 22.8 & 6.576 & 24 & 306 & 3.9 & 265 & 3.5 & 23.6 & 0.0745 & 24.8 & 0.0906 \\
\hline 3. & 4.838 & $15 \cdot 3$ & 4.379 & 16 & 236 & 2.9 & 211 & 2.8 & 20.5 & 0.0647 & 20.8 & 0.0757 \\
\hline 4. & 4.716 & 14.9 & 4.331 & 15.8 & 322 & 4.1 & 300 & 3.9 & 14.6 & 0.0462 & 14.4 & 0.0527 \\
\hline 5. & 8.048 & 25.4 & 6.939 & 25.3 & 289 & 3.7 & 293 & 3.8 & 27.8 & 0.0879 & 23.7 & 0.0864 \\
\hline 6. & 4.247 & 13.4 & 4.079 & 14.9 & 260 & 3.3 & 249 & 3.3 & 16.3 & 0.0515 & 16.4 & 0.0598 \\
\hline 7. & 5.440 & 17.2 & 5.019 & 18.3 & 375 & 4.7 & 496 & 6.5 & 14.5 & 0.0458 & 10.1 & 0.0369 \\
\hline 8. & 6.845 & 21.6 & 6.068 & 22.1 & 410 & 5.2 & 429 & 5.6 & 16.7 & 0.0527 & 14.1 & 0.0516 \\
\hline 9. & 3.603 & 11.4 & 3.396 & 12.4 & 392 & 4.9 & 377 & 4.9 & 9.2 & 0.0290 & 9 & 0.0329 \\
\hline 10. & 6.302 & 19.9 & 5.647 & 20.6 & 745 & 9.4 & 659 & 8.7 & 8.5 & 0.0267 & 8.6 & 0.0313 \\
\hline 11. & 5.588 & 17.6 & 5.024 & 18.3 & 918 & 11.6 & 846 & 11.1 & 6.1 & 0.0192 & 5.9 & 0.0217 \\
\hline 12. & 5.368 & 16.9 & 4.948 & 18.1 & 320 & 4 & 291 & 3.8 & 16.8 & 0.0529 & 17 & 0.0621 \\
\hline 13. & 2.889 & 9.1 & 2.527 & 9.2 & 245 & 3.1 & 234 & 3.1 & 11.8 & 0.0372 & 10.8 & 0.0394 \\
\hline 14. & 8.586 & 27.1 & 7.490 & 27.3 & 400 & 5.1 & 387 & 5.1 & 21.5 & 0.0677 & 19.4 & 0.0706 \\
\hline 15. & 5.382 & 17 & 4.767 & 17.4 & 690 & 8.7 & 622 & 8.2 & 7.8 & 0.0246 & 7.7 & 0.0280 \\
\hline 16. & 6.447 & 20.3 & 5.631 & 20.6 & 517 & 6.5 & 465 & 6.1 & 12.5 & 0.0393 & 12.1 & 0.0442 \\
\hline 17. & 3.285 & 10.4 & 2.951 & 10.8 & 365 & 4.6 & 348 & 4.6 & 9 & 0.0284 & 8.5 & 0.0310 \\
\hline 18. & 5.616 & 17.7 & 5.242 & 19.1 & 832 & 10.5 & 843 & 11.1 & 6.8 & 0.0213 & 6.2 & 0.0227 \\
\hline Media & 5.692 & 18 & 5.117 & 18.7 & 440 & 5.6 & 423 & 5.6 & 15.01 & 0.0473 & 14.05 & 0.0513 \\
\hline
\end{tabular}

Tabla 3.

Diccionario con los cinco vocablos más disponibles

\begin{tabular}{|c|c|c|c|c|}
\hline \multicolumn{5}{|c|}{ 1: Las partes del cuerpo } \\
\hline & \multicolumn{2}{|c|}{ Primero } & \multicolumn{2}{|c|}{ Cuarto } \\
\hline & Palabra & $\%$ Disp & Palabra & $\%$ Disp \\
\hline 1 & ojo & $61.8 \%$ & 1 ојо & $63.4 \%$ \\
\hline 2 & cabeza & $60.6 \%$ & 2 cabeza & $63.0 \%$ \\
\hline 3 & nariz & $51.9 \%$ & 3 brazo & $56.6 \%$ \\
\hline 4 & brazo & $51.7 \%$ & 4 pierna & $53.1 \%$ \\
\hline 5 & mano & $50.6 \%$ & 5 mano & $53.1 \%$ \\
\hline \multicolumn{5}{|c|}{ 3: Las partes de la casa sin los muebles } \\
\hline & \multicolumn{2}{|c|}{ Primero } & \multicolumn{2}{|c|}{ Cuarto } \\
\hline \multicolumn{2}{|r|}{ Palabra } & \% Disp & Palabra & \% Disp \\
\hline 1 & cocina & $86.8 \%$ & \multirow{5}{*}{$\begin{array}{ll}1 & \text { cocina } \\
2 & \text { salón } \\
3 & \text { baño } \\
4 & \text { habitación } \\
5 & \text { terraza } \\
\end{array}$} & $85.9 \%$ \\
\hline & salón & $75.5 \%$ & & $75.5 \%$ \\
\hline 3 & baño & $65.1 \%$ & & $64.6 \%$ \\
\hline 4 & habitación & $59.1 \%$ & & $56.6 \%$ \\
\hline 5 & terraza & $43.3 \%$ & & $45.8 \%$ \\
\hline & \multicolumn{4}{|c|}{ 5: Los alimentos y las bebidas } \\
\hline & \multicolumn{2}{|c|}{ Primero } & \multicolumn{2}{|c|}{ Cuarto } \\
\hline & Palabra & \% Disp & Palabra & $\%$ Disp \\
\hline & agua & $60.1 \%$ & 1 agua & $54.7 \%$ \\
\hline 2 & Coca-Cola* & $39.1 \%$ & 2 pescado & $29.9 \%$ \\
\hline 3 & Fanta* & $29.6 \%$ & 3 Coca-Cola* & $27.9 \%$ \\
\hline & pescado & $29.3 \%$ & 4 zumo & $27.8 \%$ \\
\hline 5 & carne & $27.8 \%$ & 5 carne & $27.3 \%$ \\
\hline
\end{tabular}

\begin{tabular}{|c|c|c|c|c|}
\hline \multicolumn{5}{|c|}{ 2: La ropa (de hombres y mujeres) } \\
\hline & \multicolumn{2}{|c|}{ Primero } & \multicolumn{2}{|c|}{ Cuarto } \\
\hline & Palabra & \multirow{2}{*}{$\begin{array}{r}\text { \% Disp } \\
73.1 \%\end{array}$} & Palabra & \% Disp \\
\hline 1 & camiseta & & 1 camiseta & $72.6 \%$ \\
\hline 2 & camisa & $65.6 \%$ & 2 pantalón & $66.4 \%$ \\
\hline 3 & pantalón & $61.7 \%$ & 3 camisa & $63.8 \%$ \\
\hline 4 & falda & $46.3 \%$ & 4 falda & $54.0 \%$ \\
\hline 5 & chaqueta & $38.5 \%$ & 5 brag(uit)a & $42.8 \%$ \\
\hline \multicolumn{5}{|c|}{ 4: Los muebles de la casa } \\
\hline & \multicolumn{2}{|c|}{ Primero } & \multicolumn{2}{|c|}{ Cuarto } \\
\hline & Palabra & $\%$ Disp & Palabra & \% Disp \\
\hline 1 & mesa & $70.8 \%$ & 1 mesa & $72.5 \%$ \\
\hline 2 & silla & $68.6 \%$ & 2 silla & $71.1 \%$ \\
\hline 3 & sofá & $56.2 \%$ & 3 cama & $61.9 \%$ \\
\hline 4 & armario & $55 \cdot 3 \%$ & 4 armario & $59.2 \%$ \\
\hline 5 & cama & $55.2 \%$ & 5 sofá & $56.5 \%$ \\
\hline \multicolumn{5}{|c|}{ 6: Objetos colocados en la mesa para la comida } \\
\hline & \multicolumn{2}{|c|}{ Primero } & \multicolumn{2}{|c|}{ Cuarto } \\
\hline & Palabra & $\%$ Disp & Palabra & $\%$ Disp \\
\hline & tenedor & $79.5 \%$ & 1 tenedor & $82.6 \%$ \\
\hline & cuchillo & $75.8 \%$ & 2 cuchillo & $79.0 \%$ \\
\hline 3 & vaso & $73.2 \%$ & 3 cuchara & $74.5 \%$ \\
\hline & cuchara & $69.5 \%$ & 4 vaso & $71.5 \%$ \\
\hline & servilleta & $62.8 \%$ & 5 servilleta & $66.3 \%$ \\
\hline
\end{tabular}




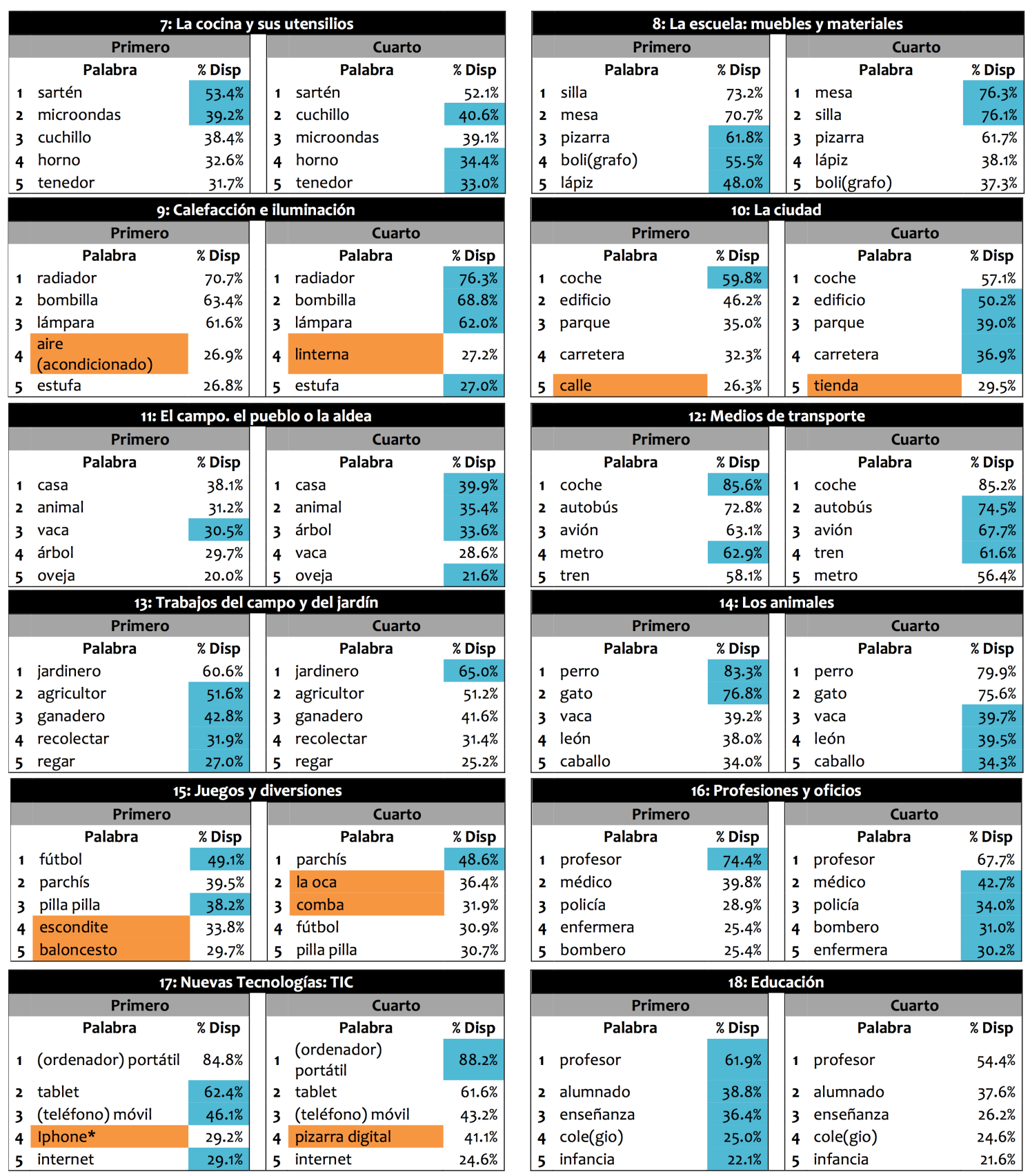

* En naranja se encuentran las palabras no compartidas

** En azul el porcentaje de disponibilidad superior para las palabras compartidas

\section{Discusión y conclusiones}

Al comenzar esta investigación la pregunta planteada era si el léxico de los futuros docentes se modificaba y/o aumentaba tras cuatro años de formación o si, por el contrario, se mantenía estable. Debido a que los trabajos clásicos de disponibilidad léxica evalúan a alumnos/as preuniversitarios para evitar la contaminación de léxica de términos específicos, inicialmente se planteó que debía existir una diferencia a favor de los alumnos/as de cuarto en relación con la disponibilidad léxica que presentaran.

Los diccionarios elaborados para esta investigación -y que tienen como objetivo ser publicados- son una contribución a la comprensión del vocabulario de los futuros profesores/as para conocer de forma detallada si se ha alcanzado una competencia 
lingüística suficiente para el desempeño de la labor docente. En estos diccionarios no se puede olvidar que no todas las entradas corresponden a una única palabra, sino que pueden presentar distintas unidades léxicas, como en el caso de pizarra digital. La presencia de estas unidades en la edición del corpus, suponen una mayor dificultad en su elaboración, pero porque se consideró que la reducción, por ejemplo a pizarra, empobrecería los diccionarios y dejaría de ser el reflejo de la realidad lingüística de los informantes.

Tras estudiar el léxico disponible evocado por los informantes de los Grados de Educación Infantil y Educación Primaria, en el comienzo (primer curso) y en el fin de sus estudios (cuarto curso), se puede concluir que para el corpus completo sí existen diferencias entre el léxico de los alumnos/as de cuarto y el de los de primero. Ahora bien, si se tienen en cuenta tan solo las palabras únicas -sin repeticiones- sólo se encuentran diferencias en los centros de interés que mantienen una relación directa con su formación en educación: el centro de interés (8) La escuela: muebles y materiales escolares y el (18) Educación. En ambas áreas asociativas los alumnos/as de cuarto emplean más palabras y más diversas que los de primero. Por lo tanto, en lo relativo a su formación directa, sí existe un cambio en el vocabulario -esto es patente en posiciones inferiores del diccionario. A su vez, relacionado también con estas áreas asociativas, se comienza a apreciar un cambio de perspectiva entre los estudiantes de cuarto y los de primero, debido a que los de cuarto emplean más palabras desde la perspectiva del profesor (quizá por verse más cercanos a esa posición y por su participación en los periodos de prácticas curriculares que ya les han mostrado la realidad de la tarea docente). Además, en estos centros de interés (el 8 y el 18) es donde se identifica una mayor variabilidad en relación con el índice de cohesión entre los alumnos/as de primero y cuarto.

Otro de los centros de interés que resultó llamativo fue el (17) Nuevas Tecnologías: TIC. En él se esperaba encontrar más terminología educativa (de distintos programas o aplicaciones educativas) que, aunque algunas de ellas como Moodle* o JClic* aparecen en posiciones inferiores y especialmente en los alumnos/as de cuarto, no muestra una diferencia clara entre ambos grupos. Esto puede deberse a la alta velocidad de cambio a la que la tecnología está expuesta y que por lo tanto, afecta a ambos grupos por igual.

El trabajo presentado no está libre de limitaciones. Las principales fueron: (1) la edición del corpus para la elaboración de los diccionarios finales, dado el alto número de palabras con las que se tuvo que trabajar, hizo que el proceso fuese lento para evitar errores. (2) Al no localizar trabajos de investigación que analizaran la variable curso académico en alumnos/as de Educación, no se pudieron comparar -en muchos momentos- los resultados obtenidos.

Finalmente, la duda que surge tras esta investigación está vinculada a cómo es posible que, tras cuatro años de formación universitaria, en la que los/las estudiantes han cursado distintas asignaturas de diferentes áreas de conocimiento, no exista una evolución o especialización más evidente del léxico -sería interesante poder compararlo con los datos de otras universidades o de otros Grados-. En todo caso, este hecho resulta relativamente preocupante, ya que serán ellos los encargados en un futuro cercano de iniciar a sus alumnos/as en el conocimiento, al ser el vocabulario que ellos empleen la puerta para cualquier futuro aprendizaje -entre otras competencias que deberán desarrollar-. Por ese motivo, se considera fundamental reforzar el léxico de los futuros docentes en las distintas áreas de conocimiento dentro del currículum universitario o, en su caso, implementar herramientas para su mejora cuantitativa y cualitativa. 


\section{Referencias}

Ávila Muñoz, A. M. (2006). Léxico disponible de los estudiantes preuniversitarios de Málaga. Málaga: Universidad de Málaga.

Bartol Hernández, J. A. (2017). Léxico panhispánico. Presentado en Jornadas de Investigación en Disponibilidad Léxica: Nuevas tendencias y aportaciones, Salamanca. Recuperado a partir de https://www.youtube.com/watch?v=1OjikY $5 Z Z C E \&$ index=1\&list=PLeKL8HuZQCJzRjn3Vda_gxedkMIT1-ZGB

Bellón Fernández, J. J. (2011). Léxico disponible de la provincia de Córdoba. España: Universidad de Las Palmas de Gran Canaria, Servicio de Publicaciones y Universidad de Córdoba, Servicio de Publicaciones.

Bellón Fernández, J. J., Samper Hernández, M., \& Samper Padilla, J. A. (2003). El proyecto de estudio de la disponibilidad léxica en español. En G. Wotjak (Ed.), Pautas y pistas en el estudio del léxico hispano(americano) (pp. 27-140). Frankfurt-Madrid: VervuertIberoamericana.

Bolaños Medina, A., \& Luján García, C. I. (2010). Análisis de los anglicismos informáticos crudos del léxico disponible de los estudiantes universitarios de traducción. Lexis: Revista de lingüística y literatura, 34(2), 241-274.

Decroly, O. (2007). La función de la globalización y la enseñanza: y otros ensayos. Madrid: Biblioteca Nueva.

Dimitrijévic, N. R. (1969). Lexical availability. A new aspect of the lexical availability of secondary school children. Heidelberg: Julius Gross Verlag.

Faria, R., Reis, P., \& Peralta, H. (2016). La formación de profesores: formación contínua o formación postgraduada? Perspectivas de profesores y de líderes de las escuelas. Revista Electrónica Interuniversitaria de Formación del Profesorado, 19(2), 289-296. Recuperado a partir de http://revistas.um.es/reifop/article/view/254891

Fernández Juncal, C. (2013). Léxico disponible en Cantabria. Estudio sociolingüístico. Salamanca: Universidad de Salamanca, Ediciones Universidad de Salamanca.

Gómez Gómez, M., \& García Aretio, L. (2016). La formación como factor clave en la integración de la Pizarra Digital Interactiva. Perspectivas de profesores y coordinadores TIC. Revista Electrónica Interuniversitaria de Formación del Profesorado, 19(3), 35-51. Recuperado a partir de http://revistas.um.es/reifop/ article/view/225451

Gómez Molina, J. R., \& Gómez Devís, M. B. (2004). La disponibilidad Léxica de los estudiantes preuniversitarios valencianos. Estudio de estratificación sociolingüística. Valencia: Universidad de Valencia.

Gougenheim, G., Michéa, R., Rivenc, P., \& Sauvageot, A. (1964). L'elaboration du français fondamental (1er degree). Étude sur l'établissement d'un vocabulaire et d'une grammaire de base. París: Didier.

Hernández Muñoz, N. (2006). Hacia una teoría cognitiva integrada de la disponibilidad léxica: El léxico disponible de los estudiantes castellano-manchegos. Salamanca: Universidad de Salamanca, Ediciones Universidad de Salamanca.

Hernández Muñoz, N. (2015). Categorías en el léxico bilingüe: perspectivas desde el priming semántico interlenguas y la disponibilidad léxica. RAEL: Revista Electrónica de Lingüística Aplicada, 13(1), 19-38. Recuperado a partir de http://www.aesla.org.es/ojs/ index.php/RAEL/article/view/21

López Chávez, J. (1994). Comportamiento sintáctico de algunos verbos ordenados según su grado de disponibilidad léxica. Revista de Estudios de Adquisición de la Lengua Española, 1, 67-84.

López Chávez, J., \& Strassburger Frías, C. (2000). El diseño de una fórmula matemática para obtener un índice de disponibilidad léxica confiable. Anuario de Letras, (38), 227-251. 
Martínez Olmos, E. (2015). El léxico disponible de las comunidades de habla alicantinas: Estudio sociolingüístico y diccionarios. Alicante: Universidad de Alicante.

Michavila, F., Martínez, J. M., \& Merhi, R. (2015). Comparación internacional del sistema universitario español. Madrid: CRUE Universidades Españolas. Recuperado a partir de http://estaticos.elmundo.es/documentos/2016/01/18/universidad.pdf

Michéa, R. (1953). Mots fréquents et mots disponibles. Un aspect Nouveau de la statistique du langage. Les Langues Modernes, 47, 338-344.

Murillo Rojas, M. (1993). Disponibilidad léxica en los preescolares: estudios de cinco campos semánticos. Kánina Revista Artes y Letras de la Universidad de Costa Rica, XVII(2), 117127.

Navarro Marrero, Y. (2009). Terminología especializada en el área de fisioterapia: acercamiento desde la metodología de la disponibilidad léxica específica. En Interlingüística XX. Barcelona: Centre de Lingüística Teòrica, Universitat Autònoma de Barcelona, Ministerio de Ciencia e Innovación, Asociación de Jóvenes Lingüístas. Recuperado a partir de http://filcat.uab.cat/clt/XXIVAJL/Interlinguistica/Encuentro \%20XXIV/Navarro_Marrero_REVF.pdf

Ortolano Ríos, B. (2005). Disponibilidad léxica de los estudiantes de Ayamonte. Interlingüística, (16), 847-857.

Quemada, B. (1967). Les Dictionnaires du français moderne: 1539-1863. París: Didier.

Ramírez García, A. (2015). La formación del profesorado de Educación Primaria ante las competencias básicas. Revista Electrónica Interuniversitaria de Formación del Profesorado, 18(3), 199-214. Recuperado a partir de http://revistas.um.es/reifop /article/view/193811

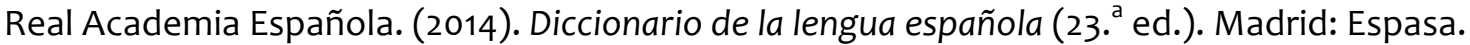

Real Academia Española, \& Asociación de Academias de la Lengua Española. (2005). Diccionario panhispánico de dudas (DPD). Madrid: Santillana.

Real Academia Española, \& Asociación de Academias de la Lengua Española. (2010). Ortografía de la lengua española. Madrid: Espasa.

Samper Padilla, J. A. (1998). Criterios de edición del léxico disponible: Sugerencias. Lingüística, 10, 311-333.

Santos Díaz, I. C. (2015). Evaluación de la competencia léxica bilingüe en estudiantes del Máster Universitario en Profesorado. Universidad de Málaga, Málaga. Recuperado a partir de https://riuma.uma.es//xmlui/handle/10630/10447

Secretaría general técnica, \& Subdirección general de estadística y estudios. (2015). Datos y Cifras. Curso escolar 2015-2016. Madrid: Ministerio de Educación, Cultura y Deporte. Recuperado a partir de http://www.mecd.gob.es/dms/mecd/servicios-al-ciudadanomecd/estadisticas/educacion/indicadores-publicaciones-sintesis/datoscifras/Datosycifras1516.pdf

Velarde Inzunza, J. de J. (2014). Análisis de léxico disponible en hablantes escolares de Culiacán, Sinaloa. Universidad Autónoma de Sinaloa, México. 


\section{Anexo 1: Prueba de disponibilidad léxica}

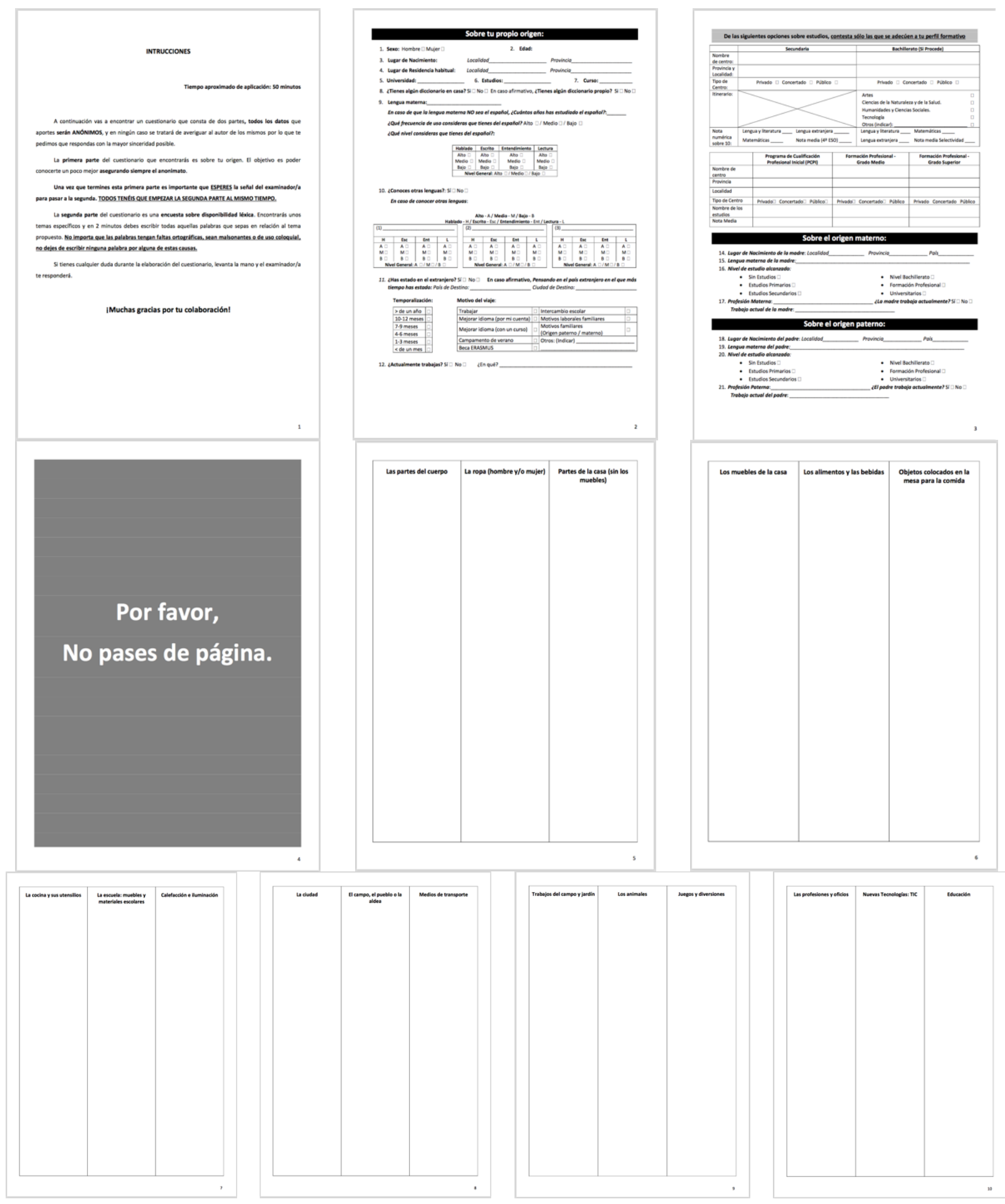

